\title{
Sleep Irregularity in the Previous Week Influences the First-Night Effect in Polysomnographic Studies
}

\author{
Da-Hye Lee ${ }^{1}$, Chul-Hyun Cho', Changsu Han', Ki-Nam Bok¹, Jung Ho Moon', \\ Eunil Lee ${ }^{2}$, Heon-Jeong Lee ${ }^{1 凶}$, and Leen Kim ${ }^{1}$ \\ ${ }^{1}$ Department of Psychiatry, Korea University College of Medicine, Seoul, Republic of Korea \\ ${ }^{2}$ Department of Preventive Medicine, Korea University College of Medicine, Seoul, Republic of Korea
}

Objective The first-night effect is a well-known phenomenon resulting from an individual's maladaptation to the unfamiliar environment of a sleep laboratory. However, there have been no direct reports of the effect of previous sleep patterns on the first-night effect. We aimed to investigate the effect the previous week's sleep pattern on the first-night effect.

Methods Twenty-four young, healthy, male participants completed the study procedure. During one week prior to study, the participants kept sleep diaries and wore actigraphs to identify sleep-wake pattern. Two consecutive nights of polysomnography were conducted after that. Wilcoxon signed-rank tests were applied to compare sleep variables of the two nights. Variance (standard deviation) of sleep onset time during the previous week was used as an index of irregularity. A Kendall's ranked correlation analysis and a linear regression test were applied to detect correlation between sleep irregularity and the first-night effect measured by polysomnography.

Results There were significant differences in the values of sleep efficiency $(\mathrm{p}=0.011)$ and wake after sleep onset (WASO) ( $\mathrm{p}=0.006)$ between the two nights. Sleep efficiency was lower and WASO was higher on the first night as compared to the second night. Sleep irregularity in the previous week was negatively correlated with sleep efficiency $(\mathrm{p}<0.001)$ of the first night, but was not significantly correlated with any other sleep parameters.

Conclusion We replicated the existence of the first-night effect commonly observed in sleep studies. Sleep irregularity in the previous week may influence the first-night effect in polysomnographic studies.

Psychiatry Investig 2016;13(2):203-209

Key Words First-night effect, Sleep irregularity, Polysomnography, Sleep efficiency.

\section{INTRODUCTION}

The first-night effect is a well-known phenomenon wherein sleep architecture is distorted during the first night in a sleep laboratory. The first-night effect is most frequently characterized by lower sleep efficiency, increased wakefulness, longer sleep and rapid eye movement (REM) sleep latency, and decreased REM sleep during initial lab recording nights compared to subsequent nights. ${ }^{1-4}$ This is thought to be due to individuals adapting to the unfamiliar surroundings of sleep

Received: March 15, 2015 Revised: June 22, 2015

Accepted: June 26, 2015 Available online: October 19, 2015

$\triangle$ Correspondence: Heon-Jeong Lee, MD, PhD

Department of Psychiatry, Korea University College of Medicine, 73 Inchonro, Seongbuk-gu, Seoul 02841, Republic of Korea

Tel: +82-2-920-6721, Fax: +82-2-929-7679, E-mail: leehjeong@korea.ac.kr

(a) This is an Open Access article distributed under the terms of the Creative Commons Attribution Non-Commercial License (http://creativecommons.org/licenses/bync/3.0) which permits unrestricted non-commercial use, distribution, and reproduction in any medium, provided the original work is properly cited. laboratory setting and the instrumentation. For many studies, it is recommended not to use data from a first "adaptation night" to control for this effect, especially in experimental setting, which can be time-consuming and expensive.

Few studies have investigated the factors influencing the first-night effect. Several factors, such as environment, age, and psychiatric illnesses of participants, have been proposed as possible causes of the first-night effect. Several studies have reported no first-night effect and negligible night-to-night variability when participants slept at home with ambulatory polysomnography in their usual surroundings. ${ }^{5-9}$ There is some evidence that older participants show a greater first-night effect. ${ }^{10,11}$ A few studies have suggested that anxiety may play a role in the first-night effect. ${ }^{12,13}$ There have been investigations of the first-night effect in psychiatric patients, such as those with major depressive disorder, ${ }^{4,14-17}$ or post-traumatic stress disorder. ${ }^{18}$ A reverse first-night effect (better sleep during the first night in a sleep laboratory) has been reported for insom- 
niacs. ${ }^{19}$ This may be because of a learned, maladaptive association between some insomniacs' attempts to sleep and heightened levels of arousal. In sum, these studies suggest that factors other than the familiarity of the environment may influence the occurrence of the first-night effect.

One factor that may influence the first-night effect is an individual's previous sleep pattern. To control for this potential effect, some studies have required that participants adhere to a regular sleep-wake pattern prior to study entry. However, to date, there have been no direct reports of the effect of previous sleep patterns on the first-night effect.

In this study, we aimed to evaluate differences in sleep architecture between the first and second night in a sleep laboratory setting to confirm the existence of the first-night effect. Furthermore we investigated the effect of the previous week's sleep pattern on the first-night effect.

\section{METHODS}

\section{Participants}

A total 30 young adult males ranging from 19 to 29 years of age (mean: $22.29 \pm 3.0$ ) were recruited for this study. We chose only young healthy male participants in order to rule out age, sex, or health-related confounds. Recruitment strategies included advertisements on Internet bulletin boards and staff referrals. All participants had no experience sleeping in experimental situations. All completed questionnaires regarding their sleeping conditions and physical and psychiatric health prior to participation. A psychiatrist (HJL) interviewed all participants before enrollment to rule out sleep, psychiatric, and medical problems. Participants underwent two consecutive laboratory-based nocturnal polysomnography (NPSG). Additionally, participants were asked not to ingest alcohol in the two weeks before and throughout the duration of the study. Six volunteers were excluded from the study for the following reasons: 1) revealing unreported sleep problems such as sleep apnea, 2) poor adherence of actigraphy and inaccurate data of sleep-wake diary, and/or 3) inadequate sleep study. A total 24 individuals participated in the study and were included in analyses. None of the participants had physical or psychiatric illnesses. Study procedures were approved by Korea University Hospital Institutional Review Boards. Participants gave written informed consent after receiving an explanation of the purpose and the procedure of the study. Participants were paid for their participation.

\section{Design}

For the week prior to NPSG, participants were instructed to adhere to regular sleep (around 11 p.m.) and wake times (around 7 a.m.) as usual. They also kept a sleep-wake diary and wore actigraphs (Actiwatch-L, Mini-Mitter) to identify sleep-wake patterns for the week. Next, two consecutive nights of polysomnography were conducted. Each participant arrived at the laboratory at 10 p.m. and slept in an individual, soundattenuated, and comfortably furnished bedroom, under the supervision of a polysomnography technician. Lights were turned off around 11 p.m. and turned on around 7 a.m. The recording lasted approximately $8 \mathrm{~h}$.

\section{Measure}

\section{Sleep questionnaire}

All participants completed a sleep questionnaire that included the Epworth Sleepiness Scale (ESS), the Athens Insomnia Scale (AIS), and the Pittsburgh Sleep Quality Index (PSQI). The ESS is a simple and brief self-administered questionnaire that measures average sleep propensity (chance of dozing) over eight situations that are commonly experienced in daily life. Propensity to fall asleep is rated on 4 -point scale $(0=$ never, $3=$ high chance of dozing). The ESS score can range from 0 to 24 , and a normal score is assumed to be 10 or less. ${ }^{20}$

The AIS is a self-assessment psychometric tool for the estimation insomnia severity encountered in a large variety of clinical and research settings. ${ }^{21}$ It consists of 8 items. Each item of the AIS can be rated $0-3,(0=$ no problem at all, $3=$ very serious problem). Two versions of this scale may be used: the entire 8-item scale (AIS-8) with a total score ranging from 0 to 24 , or a brief 5 -item version (AIS-5) with a total score ranging from 0 to $15 .{ }^{22}$ The AIS- 8 was utilized in the study and cutoff score was $6 .^{21}$ The PSQI measures self-reported sleep quality and disturbances retrospectively over the last month. ${ }^{23}$ The PSQI consists of 19 self-rated questions and five questions rated by one's bedpartner or roommate. These 19 items are grouped into 7 component scores, each weighted equally on a 0-3 scale. The sum of scores yields a global PSQI score, which can range from 0 to 21 with higher scores indicating worse sleep quality. ${ }^{24}$ Scores of 5 or more are assumed to indicate poor sleepers. ${ }^{24}$

\section{Psychiatric symptom assessments}

We used the Symptom Checklist-90-Revised (SCL-90-R) to measure participants' psychiatric symptoms. The SCL-90-R is a multidimensional, self-report symptom inventory developed by Derogatis et al., ${ }^{25}$ and translated into Korean. ${ }^{26}$ The SCL90-R consists of 90 items that are divided into 9 symptom dimensions: somatization, obsessive-compulsive, interpersonal sensitivity, depression, anxiety, hostility, phobic anxiety, paranoid ideation, and psychoticism. Each item is rated on a 5-point scale of distress, anchored by 0 (not at all) and 4 (extremely). Three global indices provide measures of overall psy- 
chological distress: the general symptom index, the positive symptom total, and the positive symptom distress index. Tscores greater than 50 are assumed to indicate significant psychiatric distress. ${ }^{26}$

\section{Sleep-wake pattern assessments}

During the week prior to NPSG, each participant kept a sleep-wake diary and wore an actigraph (Actiwatch-L, Mini Mitter) to identify sleep-wake patterns. Participants were asked to wear actigraphs all day except when taking a shower or exercising to prevent water damage. An actigraph is small portable device worn on the wrist or ankle that records movements over extended periods of time. It estimates sleep-wake schedules by raw activity scores based on computerized scoring algorithms. The American Academy of Sleep Medicine [AASM, formerly the American Sleep Disorders Association (ASDA)] introduced guidelines and reviews that have established the use of actigraphy as a reliable and valid sleep assessment method in sleep and circadian rhythms. ${ }^{27-31}$

Sleep-wake diaries are day-by-day reports of sleeping and waking activity. They are used to gather information about each participant's sleep-wake pattern. In this study, we used a sleep-wake diary that asked each participant to record following components: the time the participant went to bed; the time lights were turned off; the time the participant thinks sleep onset occurred; duration that the participant was awake after initial falling asleep; total sleep time; the time woke up; the time got out of bed; quality of sleep; start and end times wearing ac- tigraphs; the start and end times of any daytime naps; and duration in dark places.

We compared nightly data from the sleep-wake diary and the actigraph to exclude poor adherence and inaccurate data (large discrepancies). We used data from actigraphs for analysis because previous studies have suggested that an actigraph is more accurate than sleep-wake diaries. ${ }^{32,33}$

\section{Polysomnography}

All participants underwent two consecutive laboratorybased nocturnal polysomnography (NPSG) at the Sleep Disorder Clinic of Korea University Hospital in Seoul, South Korea. Sleep studies were recorded using a RemLogic (Embla, Broomfield, CO, USA). Data were recorded by using: 6-channel electroencephalography (F4-A1, C4-A1, O2-A1, F3-A2, C3-A2, O1-A2), 2-channel electrooculogram, chin electromyography, and electrocardiogram with surface electrodes. To monitor respiratory function, the following channels were included: an oronasal thermistor, a nasal pressure thermistor and pulse oximetry were used for airflow signal and calibrated transducers were for thoracoabdominal motion. Surface electromyograms from the left and right anterior tibialis were used to detect leg movements. Participants were recorded on videotape and observed continuously by a polysomnography technician. Electrode impedance was kept below $5 \mathrm{k} \Omega$. The sampling rate was $500 \mathrm{~Hz}$. Sleep stages were scored visually in 30-s epochs according to AASM guidelines. ${ }^{34}$

Table 1. Comparison of sleep parameters between the first night and the second night

\begin{tabular}{lccc}
\hline & First night (mean \pm SD) & Second night (mean \pm SD) & p-value \\
\hline Sleep efficiency (\%) & $89.15 \pm 14.21$ & $95.01 \pm 4.44$ & 0.011 \\
Sleep latency (min) & $17.27 \pm 31.76$ & $10.34 \pm 6.74$ & 0.855 \\
REM (\%) & $19.23 \pm 4.40$ & $19.25 \pm 5.02$ & 0.786 \\
RSL (min) & $107.73 \pm 59.09$ & $87.52 \pm 30.97$ & 0.191 \\
WASO (min) & $32.26 \pm 64.67$ & $11.50 \pm 18.43$ & 0.006 \\
Stage N1 (\%) & $15.20 \pm 5.59$ & $13.34 \pm 4.58$ & 0.063 \\
Stage N2 (\%) & $48.52 \pm 7.91$ & $49.96 \pm 6.17$ & 0.324 \\
Stage N3 (\%) & $17.04 \pm 7.15$ & $17.47 \pm 6.94$ & 0.558 \\
RDI & $2.79 \pm 3.31$ & $3.18 \pm 2.88$ & 0.331 \\
AHI & $1.01 \pm 1.73$ & $1.32 \pm 1.33$ & 0.061 \\
\hline
\end{tabular}

REM: rapid eye movement sleep, RSL: rapid eye movement sleep latency, WASO: wakefulness after sleep onset, RDI: respiratory distress index, AHI: apnea-hypopnea index

Table 2. Kendall's t of sleep irregularity during the previous week and differences of sleep parameters between the first and second night sleep studies

\begin{tabular}{cccccc}
\hline & $\Delta$ SE & $\Delta$ SL & $\Delta$ REM & $\Delta$ RSL & $\Delta$ WASO \\
\hline Sleep irregularity & $-0.36(\mathrm{p}=0.014)$ & $0.17(\mathrm{p}=0.264)$ & $0.22(\mathrm{p}=0.130)$ & $0.00(\mathrm{p}=1.000)$ & $0.22(\mathrm{p}=0.143)$ \\
\hline \hline : &
\end{tabular}

$\Delta$ : difference between the first night and second night sleep studies, SE: sleep efficiency, SL: sleep latency, REM: rapid eye movement sleep, RSL: REM sleep latency, WASO: wakefulness after sleep onset 


\section{Statistical analysis}

The following statistical analyses were conducted in this study. First, to determine if a first-night effect had occurred, we used a Wilcoxon signed-rank test to compare sleep variables between the two nights. Second, a Kendall's ranked correlation analysis and a linear regression test were applied to detect correlations between sleep irregularity and the firstnight effect ( $\Delta$ sleep variables: differences in sleep variables between night 1 and night 2). We regarded a variance of sleep onset time according to actigraphy as a parameter of irregular sleep pattern. The standard deviation of sleep onset time evaluated by actigraphy during the week prior to NPSG was used as an index of irregularity of the sleep-wake pattern. ${ }^{35}$ Statistical analyses were carried out using SPSS version 12.0 (SPSS Inc., Chicago, IL, USA) for Windows. Tests were considered significant when the probability value (p) was less than 0.05 .

\section{RESULTS}

Participants did not complain of any difficulties initiating or maintaining sleep, poor sleep quality, or excessive daytime sleepiness. Scores on sleep questionnaires (mean \pm standard deviation) were as follows: ESS (4.83 \pm 2.73$)$, AIS (2.5 \pm 2.11$)$, and PSQI (4.46 \pm 2.54$)$ respectively. Mean scores of psychiatric symptoms were: Somatization (40.21 \pm 2.15$)$, Obsessive-compulsive (40.75 \pm 7.39$)$, Interpersonal sensitivity (41.96 \pm 6.10$)$, Depression (39.25 \pm 4.05$)$, Anxiety (40.50 \pm 3.24$)$, Hostility (41.12 \pm 2.27$)$, Phobic anxiety (44.21 \pm 2.25$)$, Paranoid ideation (40.75 \pm 4.49$)$, Psychoticism (41.67 \pm 2.87$)$, and General symptom index (38.71 \pm 3.81 .) All of these scores were lower than the threshold score to indicate psychiatric pathology ( $\mathrm{T}$ score $<50)$.

\section{The existence of First night effect}

Forty-eight sleep studies across two consecutive nights revealed that all participants were normal sleepers without sleep disruption. Sleep efficiency reached a mean ratio of $92.08 \%$. The mean sleep latency was 13.80 min with a mean REM sleep latency of $97.63 \mathrm{~min}$. The mean percentage of different sleep stages was as follows: stage N1, 14.27\%; stage N2, 49.24\%; stage N3, 17.26\%; and REM sleep, 19.24\%. The mean wake after sleep onset (WASO) was $21.88 \mathrm{~min}$. Finally, participants showed an average of 2.98 Respiratory distress index (RDI) and 1.17 Apnea-hypopnea index (AHI).

Sleep studies revealed that there were differences in the values of sleep variables between the two consecutive nights. There were significant differences in sleep efficiency and WASO between the two nights. Sleep efficiency was lower (89.15\% versus $95.01 \%$; $\mathrm{p}=0.011$ ) and WASO was increased (32.26 min versus $11.50 \mathrm{~min} ; \mathrm{p}=0.006$ ) on the first night as compared to the second night. The sleep study of the first night also showed nonsignificant tendencies toward delayed sleep latency (17.27 min versus $10.34 \mathrm{~min}$ ) and REM sleep latency (107.73 $\mathrm{min}$ versus $87.52 \mathrm{~min}$ ) compared to the second night. A comparison of sleep parameters between the first night and second nights is presented in Table 1.

\section{Correlation between sleep irregularity and first night effect}

Correlation between sleep irregularity and sleep parameters

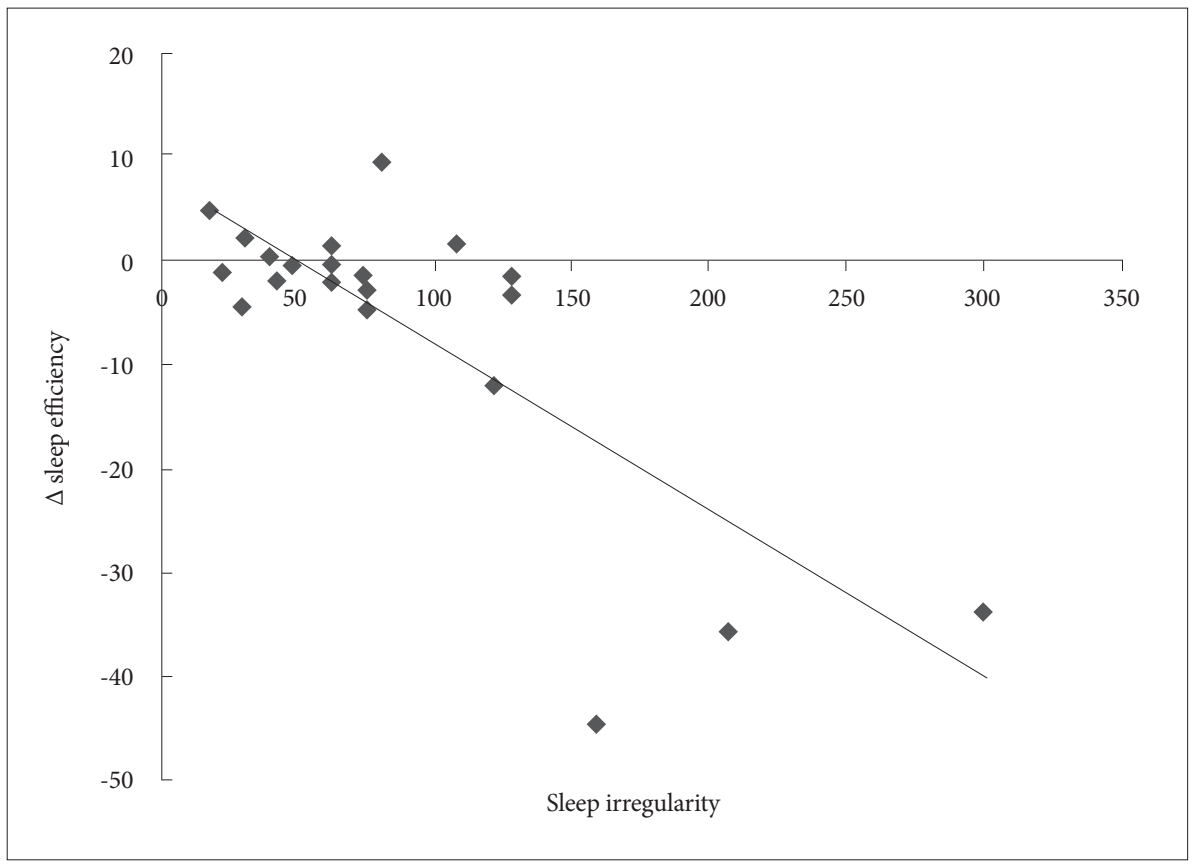

Figure 1. The linear regression shows a relationship between sleep irregularity and $\Delta$ sleep efficiency. 
is presented in Table 2. All parameters showing significant differences between consecutive nights and most reliably demonstrating first-night effects ${ }^{1-4}$ were included for analysis: sleep efficiency, sleep latency, amount of REM, REM sleep latency, and WASO. We regarded a variance of sleep onset time on actigraphy as an indicator of irregular sleep patterns during the previous week, and so standard deviation of sleep onset for each participant was used as an index of irregularity of the sleep-wake pattern. ${ }^{35}$ Sleep irregularity of the previous sleep patterns correlated with sleep efficiency (correlation coefficient: $-0.36, \mathrm{p}=0.014$ ), but did not significantly correlate with any other sleep parameters. For further statistical analysis, a linear regression test was applied to detect correlation between sleep irregularity and sleep efficiency (Figure 1). Sleep irregularity during the previous week was negatively correlated with sleep efficiency $(r=0.78, p<0.001)$.

\section{DISCUSSION}

The present study was conducted to evaluate the first-night effect and detect any relationship between previous sleep patterns and the first-night effect. We obtained mean values of sleep parameters during two consecutive nights in a sleep laboratory. We found that the first-night effect existed even in young healthy male participants and that sleep irregularity in the previous week may have a considerable influence on the first-night effect.

Our sleep study provided support for some our predictions. As expected, sleep disruption was more pronounced on the first night compared to the second night. In this regard, our data agree with previously published reports. ${ }^{1-4}$ Compared to the second night, on the first night, sleep efficiency was lower and WASO was increased significantly. Even though it was not statistically significant, the sleep study showed delayed sleep latency and REM sleep latency, and an increased proportion of stage $\mathrm{N} 1$ sleep on the night 1 as compared to night 2 . Some previous studies did not find significant differences between the first and second nights in young healthy participants with an extremely low level of psychopathology and attributed this to great adaptability in such groups. ${ }^{36,37}$ The present study showed that the first-night effect of nocturnal polysomnography exists in young healthy male participants (mean age: 22.29), as indicated by significant changes in two important sleep quality parameters: sleep efficiency and WASO. Our results are consistent with some previously published data showing that the first-night effect may still be present in young healthy participants. ${ }^{3,7,15}$ Together, these reports suggest that the first-night effect is a normal adaptive reaction of healthy participants to a new, unfamiliar sleeping environment. Therefore, we suggest that participant characteristics and environ- mental conditions may reduce but not fully eliminate the firstnight effect in sleep laboratory studies.

In addition, our sleep study provided some partial support for our predictions that sleep irregularity in the previous week may influence the first-night effect. Sleep irregularity over the previous week showed a negative correlation with sleep efficiency, but did not show significant correlations with any other sleep parameters. In reviewing these results, it is important to consider whether sleep efficiency is the most reliable sleep parameter of first-night effect or not. Previous studies have demonstrated that REM-sleep-related parameters seem to be the most sensitive and relevant to assess adaptation to the sleep laboratory across nights. 3,7,37,38 This may be caused by the slower adaptation of REM sleep to the new environment. Only a few studies have found that changes in REM sleep and sleep efficiency are good indicators of the first-night effect. ${ }^{7}$ However, results from our study showed that the first-night effect was represented by changes in sleep efficiency and WASO, not in REM sleep latency or amount of REM. This could explain that only sleep efficiency was significantly correlated with sleep irregularity in previous week. It would be possible that the association of sleep irregularity with first night effect might reflect sleep irregularity itself, not influence on first night effect. But sleep disruption was more profound on the first night compared to the second night, and it could suggest that first night effect existed rather than sleep irregularity. Therefore, we propose that sleep irregularity in the previous week influences the first-night effect because sleep irregularity is correlated with sleep efficiency, one of two parameters that represented the first-night effect in our study. Given the observed relationship between sleep irregularity and sleep efficiency, further empirical investigation of the sleep parameters that may represent the first-night effect and other previous sleep pattern indices (e.g., morningness-eveningness) that contribute to these effects seems warranted.

Although the current study provides useful information concerning the first-night effect and its related factors, there are several methodological limitations that must be noted. First, we included only 24 participants, a relatively small sample size. Second, the sample was composed of only healthy young male participants, which limits our ability to generalize findings to psychiatric patients, females, and older adults. Third, we could not control the circadian variation between participants that may be a major confounder for the first-night effect. However, our study is also unique in that it suggests that the correlation between previous sleep patterns and the firstnight effect have not yet been fully explored. Further, our study accurately identified sleep-wake patterns using both an actigraph and a sleep-wake diary. This revealed that participants did not always adhere to a regular sleep-wake pattern through- 
out the week despite of our instructions.

In conclusion, our findings corroborate previous reports that the first-night effect still occurs even when polysomnography is performed with healthy young participants in sleep laboratory with comfortable settings. ${ }^{3,7,15}$ Thus, it seems reasonable for future studies to discard data obtained from the first night of each participant's sleep assessment in order to control for this first-night effect. Because this practice is expensive and time-consuming, we had tried to find a controllable factor that predicts the first-night effect. Indeed, we have demonstrated a correlation between sleep irregularity during the previous week and sleep efficiency in the first night in the sleep laboratory. We suggest that sleep irregularity in previous week may influence the first-night effect in polysomnographic studies, and therefore, that the first-night effect may be reduced by a more regular sleep-wake pattern prior to assessments. As investigators continue to do, it is important to request that participants adhere to a regular sleep-wake pattern prior to study participation. Furthermore, our results suggest that consecutive nights of polysomnography, which are expensive and time-consuming, may not be necessary by strictly adhering to a regular sleep-wake pattern, as assessed by devices that identify sleep-wake pattern and increase adherence, such as actigraphy.

\section{Acknowledgments}

This study was supported by the Future Environmental R\&D grant funded by the Korea Environmental Industry and Technology Institute (No. RE201206020) and by the Korea Health 21 R\&D Project funded by the Ministry of Health \& Welfare, Republic of Korea (HI14C2606).

\section{REFERENCES}

1. Agnew HW Jr, Webb WB, Williams RL. The first night effect: an EEG study of sleep. Psychophysiology 1966;2:263-266.

2. Toussaint M, Luthringer R, Schaltenbrand N, Carelli G, Lainey E, Jacqmin A, et al. First-night effect in normal subjects and psychiatric inpatients. Sleep 1995;18:463-469.

3. Schmidt HS, Kaelbling R. The differential laboratory adaptation of sleep parameters. Biol Psychiatry 1971;3:33-45.

4. Mendels J, Hawkins DR. Sleep laboratory adaptation in normal subjects and depressed patients ("first night effect"). Electroencephalogr Clin Neurophysiol 1967;22:556-558.

5. Sharpley AL, Solomon RA, Cowen PJ. Evaluation of first night effect using ambulatory monitoring and automatic sleep stage analysis. Sleep 1988;11:273-276.

6. Edinger JD, Marsh GR, McCall WV, Erwin CW, Lininger AW. Sleep variability across consecutive nights of home monitoring in older mixed DIMS patients. Sleep 1991;14:13-17.

7. Browman CP, Cartwright RD. The first-night effect on sleep and dreams. Biol Psychiatry 1980;15:809-812.

8. Coates TJ, George JM, Killen JD, Marchini E, Hamilton S, Thorensen CE. First night effects in good sleepers and sleep-maintenance insomniacs when recorded at home. Sleep 1981;4:293-298.

9. Edinger JD, Fins AI, Sullivan RJ Jr, Marsh GR, Dailey DS, Hope TV, et al. Sleep in the laboratory and sleep at home: comparisons of older insomniacs and normal sleepers. Sleep 1997;20:1119-1126.

10. Webb WB, Campbell SS. The first night effect revisited with age as a variable. Waking Sleeping 1979;3:319-324.

11. Wauquier A, van Sweden B, Kerkhof GA, Kamphuisen HA. Ambulatory first night sleep effect recording in the elderly. Behav Brain Res 1991;42:7-11.

12. Riedel BW, Winfield CF, Lichstein KL. First night effect and reverse first night effect in older adults with primary insomnia: does anxiety play a role? Sleep Med 2001;2:125-133.

13. Reynolds CF 3rd, Shaw DH, Newton TF, Coble PA, Kupfer DJ. EEG sleep in outpatients with generalized anxiety: a preliminary comparison with depressed outpatients. Psychiatry Res 1983;8:81-89.

14. Kupfer DJ, Frank E, Ehlers CL. EEG sleep in young depressives: first and second night effects. Biol Psychiatry 1989;25:87-97.

15. Rotenberg VS, Hadjez J, Kimhi R, Indurski P, Sirota P, Mosheva T, et al. First night effect in depression: new data and a new approach. Biol Psychiatry 1997;42:267-274.

16. Rotenberg VS, Kayumov L, Indursky P, Hadjez J, Kimhi R, Sirota P, et al. REM sleep in depressed patients: different attempts to achieve adaptation. J Psychosom Res 1997;42:565-575.

17. Ansseau M, Kupfer DJ, Reynolds CF 3rd. Internight variability of REM latency in major depression: implications for the use of REM latency as a biological correlate. Biol Psychiatry 1985;20:489-505.

18. Woodward SH, Bliwise DL, Friedman MJ, Gusman FD. First night effects in post-traumatic stress disorder inpatients. Sleep 1996;19:312-317.

19. Hauri PJ, Olmstead EM. Reverse first night effect in insomnia. Sleep 1989;12:97-105

20. Berry RB. Sleep Medicine Pearls. Philadelphia: Hanley \& Belfus; 2003.

21. Soldatos CR, Dikeos DG, Paparrigopoulos TJ. The diagnostic validity of the Athens Insomnia Scale. J Psychosom Res 2003;55:263-267.

22. Soldatos CR, Dikeos DG, Paparrigopoulos TJ. Athens insomnia scale: validation of an instrument based on ICD-10 criteria. J Psychosom Res 2000;48:555-560.

23. Carpenter IS, Andrykowski MA. Psychometric evaluation of the Pittsburgh sleep quality index. J Psychosom Res 1998;45:5-13.

24. Buysse DJ, Reynolds CF 3rd, Monk TH, Berman SR, Kupfer DJ. The Pittsburgh Sleep Quality Index: a new instrument for psychiatric practice and research. Psychiatry Res 1989;28:193-213.

25. Derogatis LR, Rickels K, Rock AF. The SCL-90 and the MMPI: a step in the validation of a new self-report scale. Br J Psychiatry 1976;128:280289.

26. Kim K, Kim J, Won H. Korean Manual of Symptom Checklist-90-Revision. Seoul: Jung Ang Juk Sung Publisher; 1984.

27. Sadeh A, Hauri PJ, Kripke DF, Lavie P. The role of actigraphy in the evaluation of sleep disorders. Sleep 1995;18:288-302.

28. Sadeh A, Acebo C. The role of actigraphy in sleep medicine. Sleep Med Rev 2002;6:113-124.

29. Ancoli-Israel S, Cole R, Alessi C, Chambers M, Moorcroft W, Pollak $\mathrm{CP}$. The role of actigraphy in the study of sleep and circadian rhythms. Sleep 2003;26:342-392.

30. Littner M, Kushida CA, Anderson WM, Bailey D, Berry RB, Davila DG, et al. Practice parameters for the role of actigraphy in the study of sleep and circadian rhythms: an update for 2002. Sleep 2003;26:337-341.

31. Morgenthaler T, Alessi C, Friedman L, Owens J, Kapur V, Boehlecke B, et al. Practice parameters for the use of actigraphy in the assessment of sleep and sleep disorders: an update for 2007. Sleep 2007;30:519-529.

32. Vallieres A, Morin CM. Actigraphy in the assessment of insomnia. Sleep 2003;26:902-906.

33. Verbeek I, Klip EC, Declerck AC. The use of actigraphy revised: the value for clinical practice in insomnia. Percept Mot Skills 2001;92:852856.

34. Berry R, Brooks R, Gamaldo C, Harding S, Marcus C, Vaughn B. The AASM manual for the scoring of sleep and associated events: Rules, terminology and technical specifications, version 2.0. IL: J Clin Sleep Med; 2012.

35. Medeiros ALD, Mendes DBF, Lima PF, Araujo JF. The relationships between sleep-wake cycle and academic performance in medical stu- 
dents. Biol Rhythm Res 2001;32:263-270.

36. Kim EJ, Jeong DU. Investigation of'first-night effect in normal young adult male subjects on polysomnography. Sleep Med Psychophysiol 1998;5:111-117.

37. Coble P, McPartland RJ, Silva WJ, Kupfer DJ. Is there a first night ef- fect? (a revisit). Biol Psychiatry 1974;9:215-219.

38. Lorenzo JL, Barbanoj MJ. Variability of sleep parameters across multiple laboratory sessions in healthy young subjects: the "very first night effect". Psychophysiology 2002;39:409-413. 